\title{
Plasma Gelsolin Promotes Proliferation of Mesangial Cell in IgA Nephropathy
}

\author{
Lei Zhanga Dan Kong ${ }^{b}$ Hongxue Meng changsong Han ${ }^{a}$ Jiang Zhu ${ }^{d}$ \\ Juanjuan Qiao ${ }^{\mathrm{a}}$ Yan He ${ }^{\mathrm{a}}$ Tianzhen Wang ${ }^{\mathrm{a}}$ Xiaobo Li $^{\mathrm{a}}$ Fengmin Zhange \\ Xiaoming Jin ${ }^{a}$
}

aDepartment of Pathology, Basic Medical Science College, Harbin Medical University, Harbin, bepartment of Gynecology, Harbin Medical University Cancer Hospital, Harbin, 'Department of Pathology, Harbin Medical University Cancer Hospital, Harbin, dDepartment of Orthopedics, The First Affiliated Hospital of Harbin Medical University, Harbin, eDepartment of Microbiology, Basic Medical Science College, Harbin Medical University, Harbin, China

\section{Key Words}

IgA nephropathy (IgAN) - Plasma gelsolin (pGSN) - Human mesangial cells (HMCs) • Transforming growth factor beta1 (TGF- $\beta 1) \cdot$ Progression and prognosis

\begin{abstract}
Background/Aims: Plasma gelsolin (pGSN) is an actin-binding protein that plays a critical role in the pathogenesis of rheumatoid arthritis. However, whether PGSN is involved in other immunological diseases remains unknown. This study focused on the relationship between pGSN and immunoglobulin A (IgA) nephropathy (IgAN). Methods: Two hundred patients with IgAN, 200 patients each with several other types of nephropathy and healthy controls (HCs) who underwent kidney biopsies between 2000 and 2014 were enrolled in the study. The Oxford classification system was used to predict the risk of disease progression. Serum and renal tissue were used to detect pGSN, and the correlations between pGSN and IgA, galactose-deficient IgA1 (Gd-IgA1), transforming growth factor beta1 (TGF- $\beta 1$ ), fibronectin (FN) content, clinical symptoms, and kidney function were analyzed. Results: We found that the pGSN levels were significantly decreased in sera from IgAN patients compared to sera from patients with other forms of glomerular nephritis and HCs. Furthermore, the serum pGSN levels were negatively correlated with the serum IgA1, FN, and TGF- $\beta 1$ levels, and positively correlated with the estimated glomerular filtration rate. Conversely, the glomerular pGSN content was significantly elevated in the IgAN patients and was positively correlated with TGF- $\beta 1$ and FN levels. In renal tissue, the pGSN levels were significantly higher in IgAN patients with M1 and S1 compared to patients with M0 and S0 $(p<0.05)$. Meanwhile, pGSN promoted human mesangial cell (HMC) proliferation by facilitating cell mitosis in vitro. pGSN also promoted integrin $\alpha 2 \beta 1$ expression in HMCs and enhanced the integrin $\alpha 2 \beta 1-p G S N$ interaction. Conclusion: Our study suggested that pGSN may play an important role in the development of IgAN by promoting the proliferation of mesangial cells and that serum and glomerular pGSN levels may be new markers for predicting IgAN progression and prognosis.

L. Zhang, D. Kong and H. Meng contributed equally to this work.




\section{Cellular Physiology Cell Physiol Biochem 2016;40:1473-1486

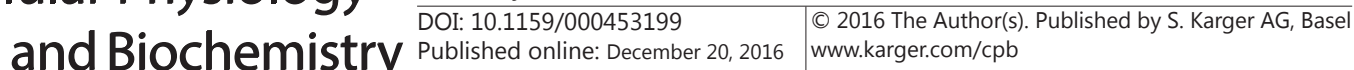 \\ Zhang et al.: The Role of pGSN in IgA Nephropathy}

\section{Introduction}

Immunoglobulin A (IgA) nephropathy (IgAN), the most common form of progressive primary glomerulonephritis (GN), is characterized by the presence of IgA complex deposition in the glomerular mesangium [1-3]. Moreover, most patients develop end-stage renal disease (ESRD) due to renal fibrosis after more than ten years. Renal fibrosis, including glomerular and tubulointerstitial fibrosis, is one of the major pathological changes that are caused by progressive IgAN [4]. Mesangial cells (MCs) play an important role in the fibrotic processes of glomerular diseases by promoting excessive synthesis and secretion of extracellular matrix (ECM). Among the ECM components, transforming growth factor beta1 (TGF- $\beta 1$ ) is a pivotal profibrotic cytokine that contributes to renal fibrosis and tubulointerstitial damage $[5,6]$. TGF- $\beta 1$ may specifically promote renal fibrosis through the downstream Smad signaling pathway [7], which can promote the mRNA expression of type I collagen and fibronectin (FN) [8], which are other important constituents of the ECM. Our study examined TGF- $\beta 1$ and FN levels to evaluate glomerular fibrosis in IgAN.

Gelsolin (GSN) is an intracellular actin-binding protein that affects cell shape, cell motility, phagocytosis and apoptosis [9]. GSN exists in both intracellular (cytoplasmic, or cGSN) and extracellular (secreted/plasma, or pGSN) forms; both of these proteins share a common antigen determinant and partial molecular homology. pGSN circulates in the blood of healthy individuals at a concentration of $200 \pm 50 \mathrm{mg} / \mathrm{L}$ and has been suggested to be a critical component of the extracellular actin-scavenging system during tissue damage [10]. Osborn [11] found that the pGSN levels in the sera of patients with rheumatoid arthritis were decreased compared to the levels in healthy controls (HCs). Furthermore, GSN-actin complexes have been detected in synovial fluids. Therefore, we hypothesized that pGSN may participate in the initiation and development of immune-related diseases. We previously found that the pGSN levels in the sera of patients with IgAN were decreased compared to those in patients with other types of glomerular nephritis and HCs, and high pGSN levels were detected in the glomeruli of IgAN patients. However, the specific role of pGSN in IgAN has remained unknown. Furthermore, the interactions between pGSN and the proliferation of MCs as well as the progression of IgAN have not previously been investigated.

The objective of the present study was to investigate the relationship between pGSN and IgAN progression and to elucidate the mechanisms of pGSN involvement in IgAN progression. Our study demonstrated that pGSN may play an important role in the development of IgAN by promoting the proliferation of MCs and that serum and glomerular pGSN levels may be markers for predicting IgAN progression.

\section{Materials and Methods}

\section{Solutions and drugs}

Fluorescein isothiocyanate (FITC)-labeled goat anti-human IgA (alpha) was purchased from KPL (Kirkegaard \& Perry Laboratories Inc. (KPL), Gaithersburg, Md.). FITC-labeled mouse anti-human IgG1 (Fc), FITC-labeled goat anti-human IgM, mouse monoclonal IgA, mouse anti-FN antibody, mouse anti-human pGSN, and rabbit anti-human GSN were purchased from Abcam Limited (Cambridgeshire, UK). FITC-labeled rabbit anti-human C3c antibody was purchased from Dako (Dako A/S, Glostrup, Denmark). Sambucus nigra agglutinin (SNA) and Vicia villosa lectin (VVL) were purchased from Vector Laboratories (Burlingame) and were used to detect serum $\alpha 2,6$-sialic acid and N-acetylgalactosamine (GalNAc), respectively. Peanut agglutinin (PNA), which was used to detect galactose (Gal), was purchased from Sigma-Aldrich (St. Louis, MO, USA). Mouse anti-human actin, mouse anti-human integrin $\alpha 2 \beta 1$ and mouse anti-human cyclin A were purchased from Santa Cruz Biotechnology, Inc. Rabbit anti-human CDK2 antibodies were obtained from Cell Signaling Technology, and FITC-conjugated goat anti-mouse IgG, Alexa Fluor ${ }^{\circledR} 594$ Goat Anti-Mouse IgG $(\mathrm{H}+\mathrm{L})$ Antibody, and peroxidase-conjugated goat anti-mouse IgG were purchased from Invitrogen (Invitrogen, Life Technologies, USA). RNA was isolated using TRIzol reagent Invitrogen, and anti-mouse IgG antibody was purchased from Vector Laboratories (Vector Laboratories, CA). An ECL Plus Detection Kit was 


\section{Cellular Physiology Cell Physiol Biochem 2016;40:1473-1486

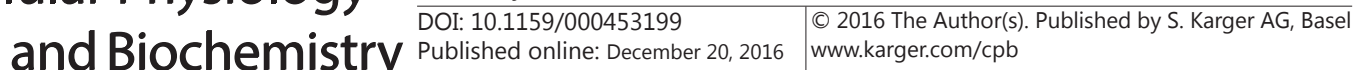 \\ Zhang et al.: The Role of pGSN in IgA Nephropathy}

purchased from Amersham Biosciences (Piscataway, NJ, USA). Recombinant human pGSN (rhpGSN) was used as a standard in the enzyme-linked immunosorbent assay (ELISA) and western blotting experiments and was purchased from Cytoskeleton, Inc. (Denver, CO, USA). Finally, the human FN ELISA kit was purchased from Wuhan Boster Bioengineering Company, Limited (China).

\section{Patients and controls}

In total, 200 patients with IgAN and 200 patients each with membranous nephropathy (MN), lupus nephritis (LN), mesangial proliferative GN (MsPGN), membrane proliferative GN (MPGN), diffuse endocapillary proliferative GN (DEPGN), focal segmental glomerulosclerosis (FSG), ischemic glomeruli (IG), minimal change glomerulopathy (MCG) and HCs underwent kidney biopsies for diagnosis. The diagnoses were performed in the Department of Pathology of Harbin Medical University from January 2010 to April 2014. Among the IgAN patients, there were 87 males and 113 females, corresponding to a male-to-female ratio of $0.77: 1$. All of the patients were between 7 and 67 years of age, with an average age of $34.72 \pm 9.36$ years. Individuals with MN (in situ immune complex deposition-induced GN) or MCG (no immune complex deposition) and HCs were sex and age matched to the IgAN patients. Serum and kidney tissues from the kidney biopsies were collected. The serum was specifically collected from the HCs during outpatient visits at the $2^{\text {nd }}$ Affiliated Hospital of Harbin Medical University. Moreover, the healthy kidney control tissues (30 cases) were collected from anatomical areas that were distant from the surgically resected kidney tumors. All of the patients and HCs provided informed consent prior to the study. Patients with diabetes, HenochSchonlein purpura or liver disease were excluded from the study. Serum pGSN levels were measured in the patients with different types of glomerular nephritis, and patients with MN or MCG and HCs were selected as the control groups for the IgAN patients in the detection of all of the studied factors in the serum and renal tissue.

\section{Oxford classification}

The renal tissues of the IgAN patients were evaluated using the IgAN Oxford classification (OC) [12]. The presence of the following findings was scored as 1 , and the absence was scored as 0 . The scoring included a mesangial (M) score of M1 or M0; a segmental glomerulosclerosis (S) score of S1 or S0; an endocapillary hypercellularity (E) score of E1 or E0; and a tubular atrophy/interstitial fibrosis (T) score of T0 ( $\leq 25 \%)$, T1 (26-50\%), or T2 (> 50\%). The MEST score was calculated as the sum of M + E + S + T.

Detection of serum pGSN, IgA, Gal-deficient IgA1 (Gd-IgA1), actin, TGF-ß1 and FN by ELISA and western blotting

Gd-IgA1 was detected using a specific lectin-binding ELISA, as previously reported [13]. Briefly, each well of a polystyrene microtiter plate (Corning, NY, USA) was coated with $5 \mu \mathrm{g} / \mathrm{mL}$ anti-human IgA in $100 \mu \mathrm{L}$ of $0.05 \mathrm{~mol} / \mathrm{L}$ bicarbonate buffer $\left(\mathrm{pH} \mathrm{9.6)}\right.$ at $4^{\circ} \mathrm{C}$ overnight. After three washes with $0.01 \mathrm{~mol} / \mathrm{L}$ phosphate-buffered saline (PBS) containing 0.05\% Tween 20 (PBS-T), the plates was incubated with 1\% fetal bovine serum (FBS) for 60 min to block their nonspecific reactivity. The plates were then incubated with serum samples from patients with IgAN, MN or MCG and HCs at room temperature for $2 \mathrm{~h}$, followed by washing with PBS-T. Next, PNA, SNA and VVL (1 mg/mL; Sigma) were diluted to 1:200 in a blocking buffer, added to each plate and incubated for $1 \mathrm{~h}$ at $37^{\circ} \mathrm{C}$, followed by 30 -min incubation at $37^{\circ} \mathrm{C}$ with horseradish peroxidase (HRP)-conjugated avidin. After a wash with PBS-T, 3, 3', 5, 5'-tetramethylbenzidine (TMB) peroxidase substrate was added, and the reaction was halted with $2 \mathrm{~mol} / \mathrm{L} \mathrm{H}_{2} \mathrm{SO}_{4}$. The absorbance was then measured with a Bio-Rad 550 microplate reader at a wavelength of $450 \mathrm{~nm}$.

Anti-human pGSN, actin and IgA were diluted to 1:1000 and were used to coat the plates, while the secondary antibody was diluted to 1:2000. The remaining steps followed the above-described methods. Human FN and TGF- $\beta 1$ ELISA kits were used to measure the FN and TGF- $\beta 1$ levels, respectively, according to the manufacturer's instructions. Of the 200 cases of each nephropathy type, 20 were randomly selected for western blotting; we specifically analyzed serum samples from 20 IgAN patients, 20 MCG patients and $20 \mathrm{MN}$ patients and $20 \mathrm{HCs}$.

The presence of pGSN in the serum was assessed by western blotting. Platelet-poor plasma was first diluted 1:20 in a sample buffer (SB) (10\% glycerol, $2 \%$ SDS, $62.5 \mathrm{mM}$ Tris- $\mathrm{HCl}, 0.03 \%$ bromophenol blue and $5 \% \beta$-mercaptoethanol, pH 6.8). rhpGSN (Cytoskeleton, Denver, CO, USA) diluted in $1 \times$ SB was then added to form a protein gradient (50 ng, $100 \mathrm{ng}, 150 \mathrm{ng}$ and $200 \mathrm{ng}$ ) and incubated at $100^{\circ} \mathrm{C}$ for $10 \mathrm{~min}$. 


\section{Cellular Physiology Cell Physiol Biochem 2016;40:1473-1486 \begin{tabular}{l|l} 
and Biochemistry & DOI: 10.1159/000453199 \\
Published online: 2016 The Author(s). Published by S. Karger AG, Basel \\
www.karger.com/cpb
\end{tabular} \\ Zhang et al.: The Role of pGSN in IgA Nephropathy}

Afterward, $20 \mu \mathrm{L}$ of each sample was subjected to 10\% SDS-PAGE, and the proteins were transferred to a PVDF membrane. After being blocked in 5\% skim milk in PBS-T for $1 \mathrm{~h}$, the membrane was incubated with an anti-pGSN antibody ( $5 \mu \mathrm{g} / \mathrm{mL}$ in $5 \%$ skim milk/PBS-T) for $1 \mathrm{~h}$. After being washed with PBS-T, the membrane was incubated with peroxidase-conjugated goat anti-mouse IgG for $1 \mathrm{~h}$, and reactive bands were detected using the ECL Plus Detection Kit. rhpGSN was used to establish a standard curve, and the pGSN levels were calculated.

Immunohistochemistry

Paraffin sections from the 200 cases of IgAN, MN, MCG and HC were pretreated with citrate $(\mathrm{pH}$ 6.0) buffer for $3 \mathrm{~min}$ at $120^{\circ} \mathrm{C}$ in a pressure cooker, and endogenous peroxidase was inhibited with $3 \%$ $\mathrm{H}_{2} \mathrm{O}_{2}$ in PBS for $10 \mathrm{~min}$. Nonspecific reactions in the sections were also blocked with goat serum for $1 \mathrm{~h}$ at room temperature. The sections were then incubated with the primary antibody anti-pGSN overnight at $4^{\circ} \mathrm{C}$, followed by incubation with the secondary antibody in 1\% BSA for $30 \mathrm{~min}$ at room temperature. The chromogenic reaction was performed via diaminobenzidine (DAB) staining, and the staining intensity was measured using appropriate software (ACT-1, Nikon).

All frozen sections were blocked with 5\% normal goat serum in PBS (pH 7.4). IgA, IgG, IgM and the complement component C3 were detected in human kidney tissues using FITC-labeled anti-human IgA, IgG, IgM and C3 antibodies (1:100), respectively. Additionally, the FN and TGF- $\beta 1$ levels were measured using an indirect fluorescence detection method. For this purpose, the tissue sections were incubated with human monoclonal anti-FN and anti-TGF- $\beta 1$ (1:100 dilution for $12 \mathrm{~h}$ at $4^{\circ} \mathrm{C}$ ), washed three times in PBS and incubated with FITC-labeled anti-mouse IgG antibody. The total number of glomeruli per slice was counted under $400 \times$ magnification in randomly chosen images that were acquired using a fluorescence microscope (Nikon E800), a digital camera (Nikon 1200F) and accompanying software (ACT-1, Nikon).

Moreover, human mesangial cells (HMCs) with or without pGSN treatment (10 mg/L) were harvested, and microscope slides were prepared. After fixation in 4\% paraformaldehyde, the cells were blocked with goat serum for $30 \mathrm{~min}$ at room temperature. Next, mouse anti-human integrin $\alpha 2 \beta 1$ antibody was diluted with 1:50 in PBS and added to the cells at $4^{\circ} \mathrm{C}$ for $14 \mathrm{~h}$. After washing with PBS, rabbit anti-mouse secondary antibody labeled with HRP was diluted $1: 100$ and added to the cells at $37^{\circ} \mathrm{C}$ for $30 \mathrm{~min}$. Finally, after washing with PBS and mounting with 10\% glycerol, the cells were observed under a microscope (OLYMPUS CX31), and images were acquired.

\section{Isolation of serum IgA1 in patients with IgAN}

IgA1 was isolated from the pooled sera of 20 patients by Jacalin affinity chromatography. The procedures were performed according to the protocol of Wang and Diven $[14,15]$. Briefly, the pooled sera were diluted 1:1 with PBS (pH 7.1) and centrifuged at $2000 \mathrm{rpm}$ for $8 \mathrm{~min}$. Then, $3 \mathrm{~mL}$ of the supernatant from each sample was loaded onto a Jacalin affinity column. The column was prepared with Jacalin immobilized on cross-linked 4\% agarose beads with an IgA1-binding capacity of $2 \mathrm{mg} / \mathrm{mL}$ gel. After the pooled serum samples were incubated on the Jacalin affinity column for $30 \mathrm{~min}$, the column was washed with $175 \mathrm{mM}$ Tris-HCl (pH 7.4) until the optical density (OD) at $280 \mathrm{~nm}$ was less than 0.10 . The IgA1 was then eluted with 0.15 M melibiose (Yuanye Biotechnology Limited Company, Shanghai, China) in $175 \mathrm{mM}$ Tris- $\mathrm{HCl}$ in $3.0 \mathrm{~mL}$ fractions until the OD returned to 0.1. After dialysis, identification and freeze drying of the Jacalin-binding proteins (from the IgAN patients), $2 \mathrm{mg} / \mathrm{mL}$ samples were separated by molecular sieve chromatography using a $2.0 \times 57 \mathrm{~cm}$ Sephacryl S-200 HR column in a Pharmacia Smart System (ÄKTAprime plus) equipped with a micropeak detector (Pharmacia Biotech, Uppsala, Sweden). The Sephacryl S-200 HR column was then eluted with buffer solution, and three distinct peaks in the OD280 absorption profile were obtained: poly IgA1 (pIgA1); monomeric IgA1 (mIgA1); and other, non-IgA1 protein. The mIgA1 protein concentrations in the samples from the IgAN patients were determined using a BCA kit (BiYuntian Biotechnology Research Institute, Shanghai, China) according to the manufacturer's instructions. The molecular weight of the mIgA1 fraction was determined by SDS-PAGE and western blot analysis using mouse anti-human IgA1 antibody. To prepare aggregated IgA1 (aIgA1), the samples of identified mIgA1 were heated at $63^{\circ} \mathrm{C}$ for $150 \mathrm{~min}$. The molecular weight of the aIgA1 fraction was also determined by SDS-PAGE, and the purity of the aIgA1 was calculated as the ratio of the aIgA1 concentration to the total protein concentration at a level of $95 \%$ using a BCA kit. The aIgA1 products were then freeze dried using an ultra-freeze-drying instrument for $24 \mathrm{~h}$ to improve their concentration for subsequent experiments. 


\section{Cellular Physiology Cell Physiol Biochem 2016;40:1473-1486 \begin{tabular}{l|l|l} 
and BiOChemistry & Dublished online: December 20, 2016 & $\begin{array}{l}\text { (c) 2016 The Author(s). Published by S. Karger AG, Basel } \\
\text { www.karger.com/cpb }\end{array}$
\end{tabular} \\ Zhang et al.: The Role of pGSN in IgA Nephropathy}

Cell culture and cell viability assay

HMCs were purchased from Cobioer Biosciences Co., LTD (China) and cultured at $37^{\circ} \mathrm{C}$ and $5 \% \mathrm{CO}_{2}$ in RPMI 1640 medium containing FBS (10\%), penicillin $(100 \mathrm{U} / \mathrm{mL})$, glutamine $(2 \mathrm{mmol} / \mathrm{L})$, and streptomycin $(100 \mu \mathrm{g} / \mathrm{mL})$. The proliferation of the HMCs was determined using Cell Counting Kit-8 (CCK-8; SigmaAldrich, St. Louis, MO) according to the manufacturer's instructions. HMCs were seeded on 96 -well culture plates at $5 \times 10^{3}$ cells/well and then incubated with $10 \mathrm{mg} / \mathrm{L} \mathrm{pGSN}$ for $24 \mathrm{~h}, 48 \mathrm{~h}, 72 \mathrm{~h}$ or $96 \mathrm{~h}$. Next, $10 \mu \mathrm{L}$ WST-8 was added to each well and incubated for $4 \mathrm{~h}$ at $37^{\circ} \mathrm{C}$, after which the absorbance was measured on a spectrophotometer/microplate reader (Bio-Rad) at a wavelength of $450 \mathrm{~nm}$. Cells cultured without pGSN were used as a control.

\section{Cell synchronization and cell cycle phase determination}

To observe the effect of the cell cycle on growth after pGSN treatment, subconfluent cultures of HMCs were synchronized in the G2/M phase using colchicines. In particular, cells $\left(10^{5}\right)$ were incubated on 9.6- $\mathrm{cm}^{2}$ disks in RPMI 1640 containing $10 \%$ FBS in a $\mathrm{CO}_{2}$ incubator at $37^{\circ} \mathrm{C}$ for $12 \mathrm{~h}$, after which colchicines $(0.1$, $0.2,0.5,0.8$ or $1 \mu \mathrm{g} / \mathrm{mL}$ ) were added to the cells for $12 \mathrm{~h}$ or $24 \mathrm{~h}$. Adherent cells were collected by treatment with trypsin and then washed with pre-chilled PBS. The cells were then fixed in $2 \mathrm{~mL}$ of cold $75 \%$ ethanol overnight at $4^{\circ} \mathrm{C}$ and resuspended in staining buffer $(20 \mu \mathrm{g} / \mathrm{mL}$ propidium iodide (PI; Sigma), $0.1 \%$ Triton $\mathrm{X}-100$ and $0.2 \mathrm{mg} / \mathrm{mL}$ RNase in PBS) for $2 \mathrm{~h}$ at $4^{\circ} \mathrm{C}$. The PI-stained cells were analyzed using FACS (FACScan; $\mathrm{BD}$ ), with at least $10^{5}$ cells counted for each sample. A data analysis was performed using CXP Software (Beckman Coulter, Inc.). The cell cycle phase was also detected in HMCs treated with pGSN (10 mg/L) at 3 h, 6 h, 9 h or $12 \mathrm{~h}$.

\section{Co-immunoprecipitation}

HMCs were treated with pGSN alone or with pGSN and aIgA1 or left untreated for $48 \mathrm{~h}$. Proteins were extracted using lysis buffer (10 mM Tris, pH 7.5; $100 \mathrm{mM} \mathrm{NaCl;} 5 \mathrm{mM}$ EDTA; $1 \%$ Triton X-100; and $0.05 \%$ SDS with complete protease inhibitor). Protein samples were then immunoprecipitated with $2 \mu \mathrm{g}$ of rabbit anti-human GSN and mouse anti-human integrin $\alpha 2 \beta 1$ antibodies at $4^{\circ} \mathrm{C}$ overnight, after which the immunoprecipitated proteins were pulled down with Protein A/G Mix Magnetic Beads (protein A-agarose beads; Merck KGaA, Darmstadt, Germany) at $4^{\circ} \mathrm{C}$ for $4 \mathrm{~h}$. The immunoprecipitates were subsequently boiled in $2 \times$ SDS loading buffer and analyzed by western blotting.

\section{Statistical analysis}

The data are presented as the means \pm standard errors of the mean. The data were analyzed using SPSS 13.0 (SPSS Inc., Chicago, IL, USA). A statistical analysis of the differences between the serum pGSN levels and the other clinical data was performed using the Mann-Whitney U-test. Pearson's correlation was used to evaluate all correlations. Moreover, two-way ANOVA was used to evaluate the cell proliferation level, and significant differences among the different grades of the Oxford classification were calculated using the unpaired t-test. Significance was defined as $p<0.05$.

Ethics

This study was approved by the Ethics Committee of Harbin Medical University. All of the individuals who were included in the study provided informed consent.

\section{Results}

pGSN levels in patients with several types of glomerular nephritis and in HCs

The pGSN levels were significantly decreased in the sera of patients with IgAN compared to those in patients with IG or MCG and in HCs, as determining using an ELISA and western blot assay ( ${ }^{* *} p<0.01$, Fig. 1A; $p<0.05$, Fig. 1B, 1C, 1D). Moreover, the pGSN levels were significantly decreased in the sera of patients with MN, LN, MPGN, MsPGN, DEPGN or FSG compared to the levels in patients with IG or MCG and in HCs $\left({ }^{*} p<0.05\right.$, Fig. $\left.1 \mathrm{~A}\right)$. 


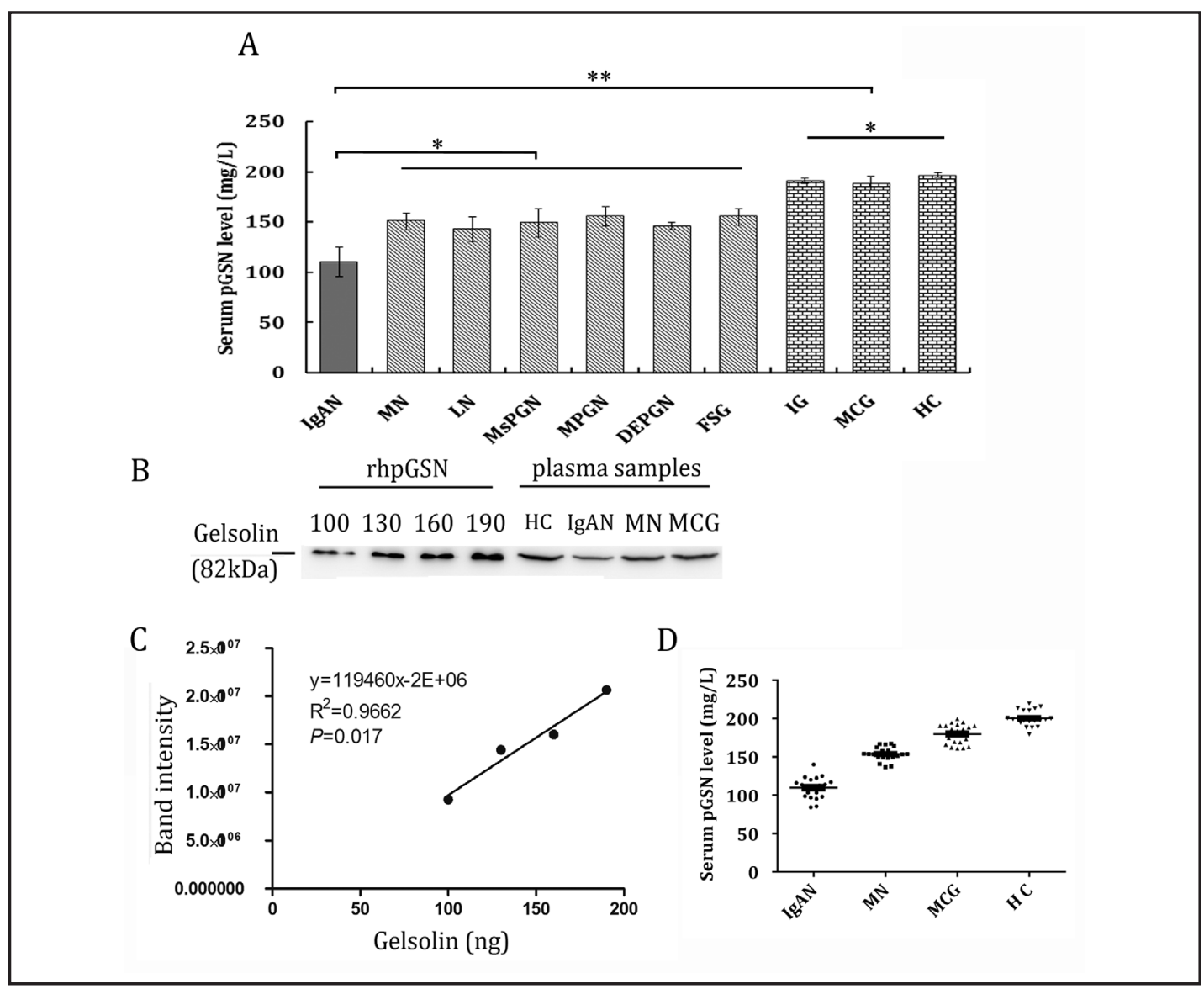

Fig. 1. ELISA and western blot detection of pGSN in the serum. (A) Serum pGSN in patients, as assessed by ELISA. (A) The serum pGSN levels were reduced in IgAN patients compared to patients with other nephropathies and HCs. There were significant differences in the pGSN levels between the IG and MCG patients and the HCs $\left({ }^{* *} p<0.01\right)$, and there were moderate differences in the pGSN levels among the MN, LN, MsPGN, MPGN and DEPGN patients $\left({ }^{* *} p<0.05\right)(\mathrm{n}=200)$. (B and C). Serum pGSN was assessed in IgAN patients by western blotting. (B, C) Representative semiquantitative western blot depicting the standard curve for the determination of the pGSN concentration in a typical experiment. (B) Lanes 1, 2, 3 and 4 contained 100, 130, 160 and $190 \mathrm{mg} / \mathrm{L}$ rhpGSN, respectively. The serum pGSN in lanes 5 was from an HC, and the serum pGSN in lanes 6, 7, and 8 was from IgAN, MN and MCG patients, respectively. (C) The 'integrated intensity' of each band on the western blot minus the adjacent background signal was plotted versus known amounts of GSN and fitted to a straight line $\left(\mathrm{n}=4, p=0.017, \mathrm{r}^{2}=0.9662\right)$. (D) The serum pGSN levels in the IgAN patients were significantly lower than those in the HCs $\left({ }^{*} p<0.05\right)$. The total number of patients in each group (n = 20) was used for statistical analysis. Note: Membranous nephropathy (MN), lupus nephritis (LN), membrane proliferative GN (MPGN), mesangial proliferative GN (MsPGN), diffuse endocapillary proliferative GN (DEPGN), focal segmental glomerulosclerosis (FSG), ischemic glomeruli (IG), minimal change glomerulopathy (MCG), and healthy control (HC).

The serum concentrations of actin, IgA, Gd-IgA1, FN and TGF- $\beta 1$, as measured using ELISA, in patients with IgAN, MN or MCG and in HCS

The serum IgA, FN, TGF- $\beta 1$ and Gd-IgA1 levels in patients with IgAN were higher than those in the control groups $\left({ }^{* *} p<0.01,{ }^{* *} p<0.01,{ }^{*} p<0.05\right.$ and ${ }^{*} p<0.05$, respectively), namely, the MCG and HC groups (Fig. 2A1, 2A2, 2A3 and 2A4, respectively). However, there was no difference in the actin levels among the groups (data not shown). In the IgAN patients, the serum pGSN levels were negatively correlated with the serum IgA levels (Fig. 2B1, $r$ $=-0.2231,{ }^{*} p=0.0165$ ). In addition, the serum FN and IgA levels were strongly positively correlated in the IgAN patients (Fig. $2 B 2, r=0.4994,{ }^{* *} p<0.0001$ ). Finally, the serum pGSN 


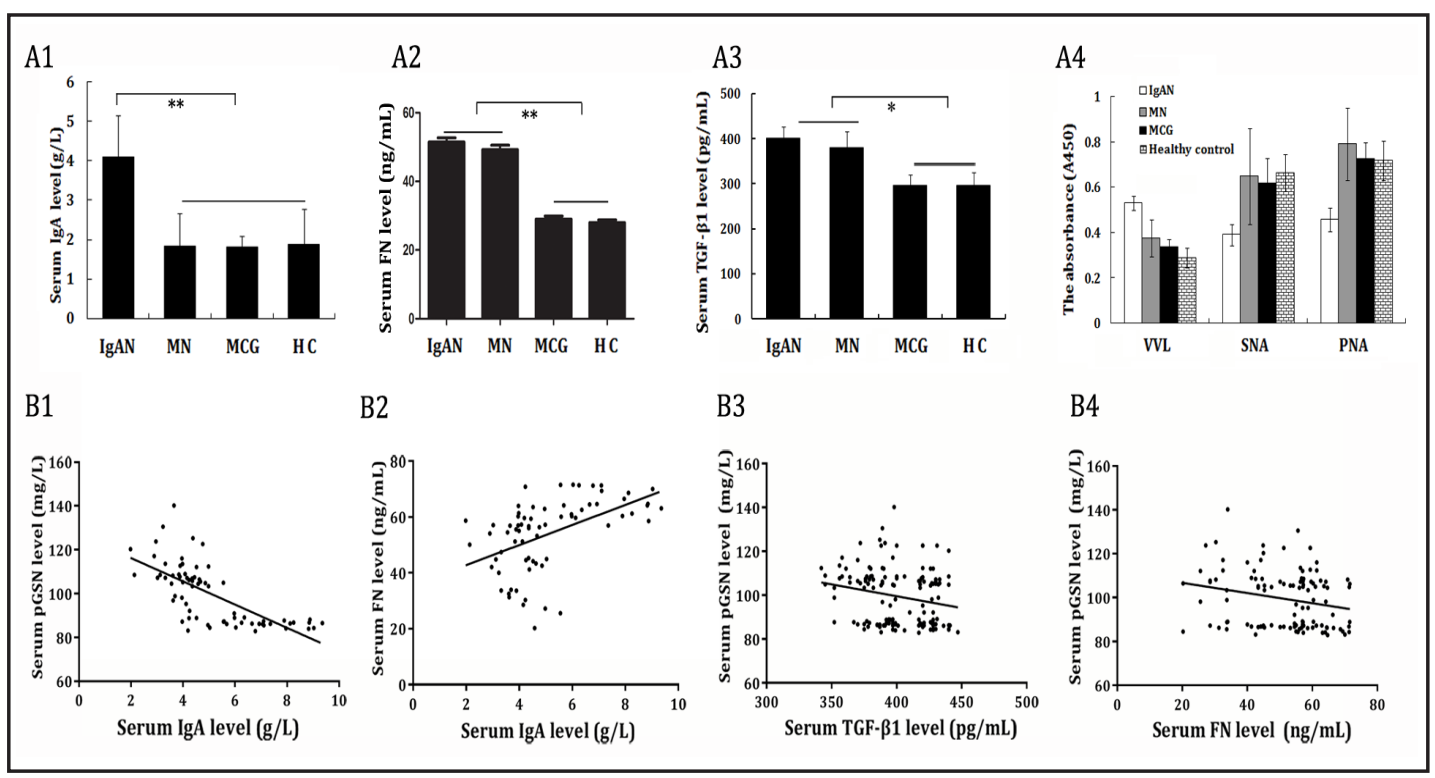

Fig. 2. (A) Serum IgA, FN, TGF- $\beta 1$ and Gd-IgA1 levels in IgAN, MN and MCG patients and HCs. (A1) Serum IgA levels in each group. The serum IgA levels in the IgAN patients were higher than those in the other groups $\left.{ }^{* *} p<0.01\right)$. (A2) Serum FN levels in each group. The serum FN levels in the IgAN patients were higher than those in the other groups $\left({ }^{* *} p<0.01\right)$. (A3) Serum TGF- $\beta 1$ levels in each group. The serum TGF- $\beta 1$ levels were higher in the IgAN and MN patients than in the MCG patients and HCs $\left(^{*} p<0.05\right.$ ). (A4) Serum IgA1 levels in each group. The serum IgA1 levels in the IgAN patients were higher than those in the other groups $\left({ }^{*} p<0.05\right)$. (B) Correlations between serum pGSN and IgA, FN and TGF- $\beta 1$. (B1) Relationship between pGSN and IgA1. The scatter diagram shows a negative correlation between pGSN and IgA $\left(r=-0.2231,{ }^{*} p\right.$ $=0.0165)$. (B2) The serum FN and IgA levels were strongly positively correlated in the IgAN patients $(\mathrm{r}=$ $\left.0.4994,{ }^{* *} p<0.0001\right)$. (B3) Relationship between pGSN and TGF- $\beta 1$. The scatter diagram shows a significant negative correlation between pGSN and TGF- $\beta 1\left(\mathrm{r}=-0.2300,{ }^{*} p=0.0134\right)$. (B4) Relationship between pGSN and FN. The scatter diagram shows a significant negative correlation between pGSN and FN $\left(\mathrm{r}=-0.2141,{ }^{*} p\right.$ $=0.01$ ). The correlations between results were evaluated using Pearson's correlation. $p<0.05$ was considered as significant. The total number of patients in each group $(n=200)$ was used for statistical analysis.

levels were strongly negatively correlated with TGF- $\beta 1$ and FN in the IgAN patients (Fig. 2B3, $r=-0.2300,{ }^{*} p=0.0134$; Fig. $\left.2 \mathrm{~B} 4, \mathrm{r}=-0.2141,{ }^{*} p=0.01\right)$. However, there was no correlation between actin and pGSN in this group $(p=0.4352)$.

\section{pGSN, TGF- $\beta 1$ and FN expression in renal tissue in IgAN}

pGSN deposition in the tissues from the IgAN and MN patients was significantly higher than that in the tissues from the MCG patients and the HCs $\left({ }^{*} p<0.05\right.$, Fig. 3A1). In contrast, there was no difference in pGSN deposition between the MN and IgAN tissues. There were also no differences in actin expression among the IgAN, MN, MCG and HC groups. The IgA fluorescence in the IgAN group was stronger than in the MN, MCG and HC groups ${ }^{* *} p<0.01$, Fig. 3A2). pGSN was mainly deposited in the area of mesangial proliferation of the glomeruli or the epithelial cells of the tubules in IgAN (Fig. 3B1-3B4). The pGSN levels in the renal tissue were not significantly higher in IgAN patients with E1 and T1 compared to those with E0 and T0 (Table 1, Fig. 3B5, $p>0.05$ ) but were significantly higher in IgAN patients with M1 and $\mathrm{S} 1$ compared to those with M0 and S0 (Table 1 , Fig. $3 \mathrm{~B} 5,{ }^{*} p<0.05$ ).

TGF- $\beta 1$ and FN were mainly expressed in the glomeruli and were weakly expressed in the tubules (Fig. 4A-4D). The pGSN levels and both the TGF- $\beta 1$ and the FN levels in renal tissues were positively correlated (Fig. $4 \mathrm{E}, 4 \mathrm{~F}, \mathrm{r}=0.2183,{ }^{*} p=0.0191$ and $\mathrm{r}=0.1851,{ }^{*} p=$ 0.0477, respectively).

\section{KARGER}


A1

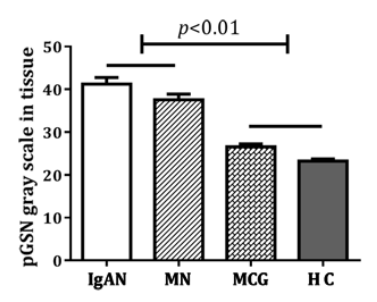

B

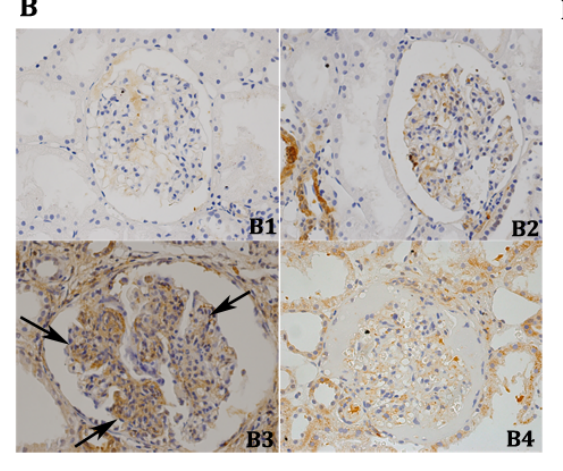

A2

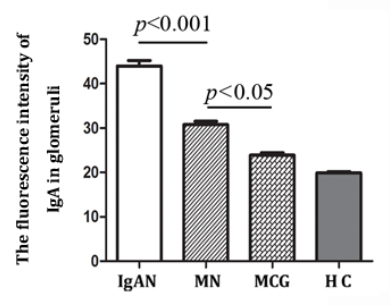

B5

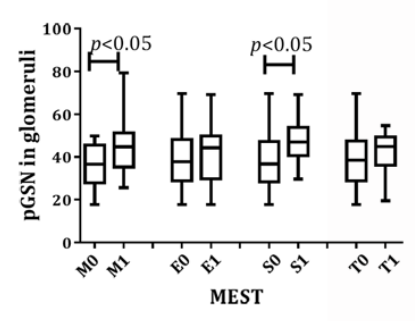

Fig. 3. (A) Deposition of pGSN and IgA in kidney tissue. (A1) pGSN deposition in the glomeruli. pGSN frequently appeared in the glomeruli in the IgAN samples. The deposition of pGSN in kidney tissue in the IgAN group was greater than that observed in the other groups $\left({ }^{*} p<0.01\right)$. (A2) Fluorescence intensity of IgA in the glomeruli. The deposition of IgA in the glomeruli in the IgAN group was stronger than that in the other groups $\left({ }^{* *} p<0.01\right)$. The difference between the results was evaluated using t-tests; $p<0.05$ was considered significant. The total number of patients in each group $(n=200)$ was used for statistical analysis. (B) pGSN deposition in the glomeruli in IgAN. pGSN was mainly deposited in the glomeruli and tubules. (B1 and B2) A small amount of pGSN was expressed in the normal glomerulus and in the epithelium of the distal convoluted tubule. (B3) pGSN was mainly expressed in the area of mesangial proliferation (arrow) in the glomerular and renal tubular epithelial cells. (B4) The deposition of pGSN in fibrotic glomeruli was lower than that in the area of mesangial proliferation of the glomeruli. (B5) The deposition of pGSN in the glomeruli in IgAN increased significantly in M1 and S1 compared to M0 and S0 $\left({ }^{*} p<0.05\right)$. The differences between results were evaluated using t-tests; $p<0.05$ was considered significant. The total number of patients in each group $(\mathrm{n}=200)$ was used for statistical analysis.

Correlations between the serum and renal tissue pGSN levels and the clinicopathological data of IgAN patients

Serum and kidney pGSN levels were negatively correlated (Fig. 5A, $r=-0.2733$, ${ }^{*} p=0.0031$ ), whereas serum pGSN levels and the estimated glomerular filtration rate (eGFR) were positively correlated (Fig. 5B, Table 2, $\mathrm{r}=0.3033,{ }^{*} p=0.0126$ ). However, there were no correlations between the serum $\mathrm{BUN}$ and $\mathrm{SCr}$ values, $\mathrm{CO}_{2} \mathrm{CP}$, or the 24-h urine protein level and pGSN levels (Table 2, $\mathrm{r}=-0.2394, p=0.0510 ; \mathrm{r}=-0.207$, $p=0.0954 ; \mathrm{r}=-0.05544, p=0.6559 ;$ and $\mathrm{r}=$
Table 1. Relationship between pGSN in renal tissue and pathologic variables of IgAN

\begin{tabular}{llll}
\hline & $P$ value & MEST & pGSN (gray scale) \\
\hline M & ${ }^{*} p<0.05$ & M0 (n=61) & $38.545 \pm 11.956$ \\
& & M1 $(n=139)$ & $47.904 \pm 10.103$ \\
E & NS & E0 (n=99) & $38.758 \pm 12.248$ \\
& & E1 $(n=101)$ & $42.209 \pm 11.946$ \\
S & \multirow{2}{*}{$p<0.05$} & S0 (n=144) & $37.682 \pm 12.128$ \\
& & S1 $(n=56)$ & $47.803 \pm 8.889$ \\
T & NS & T0 (n=162) & $39.461 \pm 11.823$ \\
& & T1 $(n=38)$ & $40.971 \pm 12.363$ \\
\hline
\end{tabular}




\section{Cellular Physiology Cell Physiol Biochem 2016;40:1473-1486 \begin{tabular}{ll|l} 
DOI: 10.1159/000453199 & $\begin{array}{l}\text { O 2016 The Author(s). Published by S. Karger AG, Basel } \\
\text { www.karger.com/cpb }\end{array}$
\end{tabular} \\ Zhang et al.: The Role of pGSN in IgA Nephropathy}

Table 2. Relationships between the serum pGSN levels and clinicopathological data of IgAN patients. (a) Reference value for hypertension: $>140 / 90 \mathrm{mmHg}$, (b) Reference value for BUN: 2.9-8.2 $\mathrm{mmol} / \mathrm{L}$, (c) Reference value for Scr: 45-104 $\mathrm{mmol} / \mathrm{L}$, (d) Normal range of $\mathrm{CO}_{2} \mathrm{CP}: 22$ $30 \mathrm{mmol} / \mathrm{L}$, (e) 24-h urinary protein $\geq 0.15 \mathrm{~g}$, (f) eGFR $(\mathrm{mL} / \mathrm{min} / 1.73 \mathrm{~m} 2)$

\begin{tabular}{|c|c|c|}
\hline Clinicopathological data & $\mathrm{n}=200(\%)$ & $\begin{array}{l}\text { Correlations between the serum pGSN } \\
\text { level and IgAN clinicopathological variables }\end{array}$ \\
\hline Symptom before seeing a doctor & Cases $(\%)$ & Mann-Whitney U test \\
\hline Edema & $152(76)$ & $p=0.013^{*}$ \\
\hline Flu and fever & $99(49.5)$ & $p=0.636$ \\
\hline Pharyngalgia and antiadoncus & $85(42.5)$ & $p=0.177$ \\
\hline Gross hematuria & $61(30.5)$ & $p=0.663$ \\
\hline a)Hypertension & $31(15.5)$ & $p=0.899$ \\
\hline Kidney function change & & Spearman correlation analysis \\
\hline b)BUN & & $r=-0.2394, p=0.0510$ \\
\hline c) $\mathrm{SCr}$ & & $r=-0.207, p=0.0954$ \\
\hline d) $\mathrm{CO}_{2} \mathrm{CP}$ & & $\mathrm{r}=-0.05544, p=0.6559$ \\
\hline e)24-h urine protein level & & $r=-0.1392, p=0.1379$ \\
\hline f)eGFR & & $\mathrm{r}=0.3033,{ }^{*} p=0.0126$ \\
\hline
\end{tabular}

Fig. 4. Deposition of TGF- $\beta 1$ and FN in renal tissue and correlations between TGF- $\beta 1$ and $\mathrm{FN}$ in the glomerulus and pGSN in the glomerulus. (A-B) TGF- $\beta 1$ expression in renal tissue in HCs and IgAN (400× magnification). A. TGF- $\beta 1$ was negatively expressed in normal glomeruli and tubules. (B) TGF- $\beta 1$ was mainly deposited in the glomeruli or the epithelia of the tubules in IgAN, and especially in the area of mesangial proliferation. (C-D) FN expression in renal tissue in HCs and IgAN. FN was mainly deposited in the glomeruli or the epithelia of the tubules in IgAN. C. FN was weakly expressed in normal glomeruli and tubules. D. FN was mainly deposited in the glomeruli or the epithelia of the tubules in IgAN. (E-F) Analysis of the correlations between TGF- $\beta 1$ and FN in the glomerulus and pGSN in the glomerulus. (E) TGF- $\beta 1(r$ $\left.=0.2183,{ }^{*} p=0.0191\right)$. $(\mathrm{F}) \mathrm{FN}(\mathrm{r}=0.1851$, $\left.{ }^{*} p=0.0477\right)$. The correlations between the results were evaluated using Pearson's correlation. $p<0.05$ was considered significant. The total number of patients in each group $(n=200)$ was used for statistical analysis.
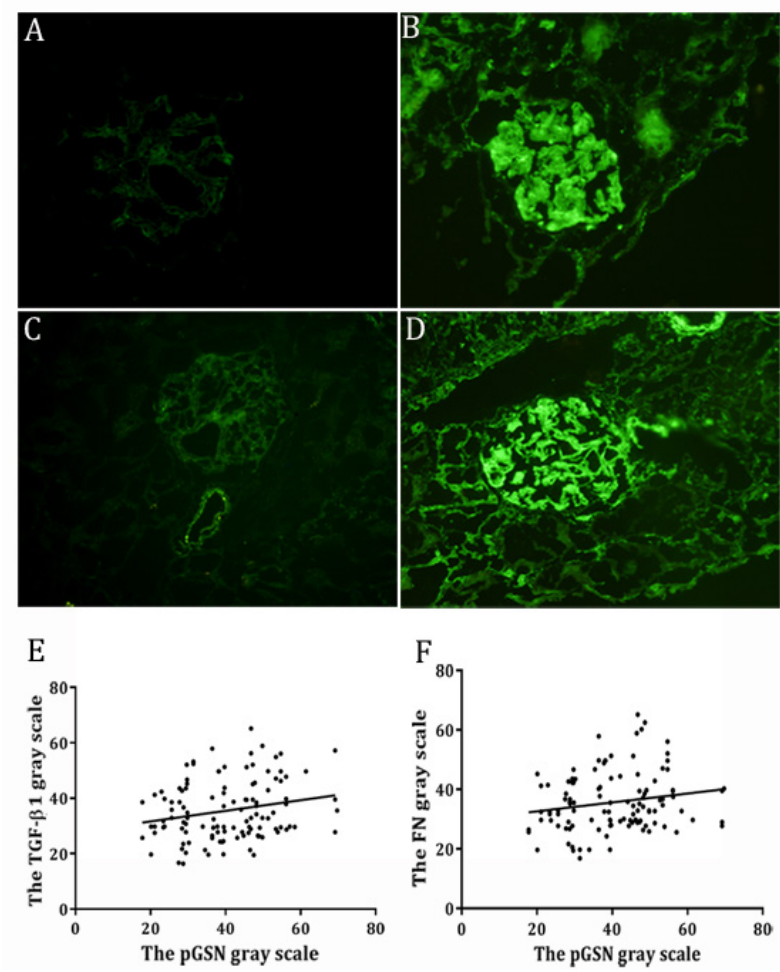

$-0.1392, p=0.1379$, respectively). The relationships between the pGSN concentration and major clinical symptoms (e.g., upper respiratory tract infection, sore throat, hypertension, edema and gross hematuria) were also analyzed (Table 2). There was a significant difference in the serum pGSN levels between patients with and without edema (Mann-Whitney U-test, $\left.{ }^{*} p=0.013\right)$.

\section{Correlations between $p G S N$ and HMC proliferation}

We chose two different time points ( 24 and $48 \mathrm{~h}$ ) at which to add $10 \mathrm{mg} / \mathrm{L}$ pGSN to stimulate MC proliferation. pGSN promoted the proliferation of HMCs in vitro, especially at $48 \mathrm{~h}$ (Fig. 6A, two-way ANOVA, $p<0.05$ ). Cell cycle phases were also detected in the MCs that were treated with pGSN (10 mg/L) at $3 \mathrm{~h}, 6 \mathrm{~h}, 9 \mathrm{~h}$ or $12 \mathrm{~h}$. After pGSN treatment for $6 \mathrm{~h}$, the percentage of cells in the $\mathrm{S}$ phase of the cell cycle increased, while the percentage 
A

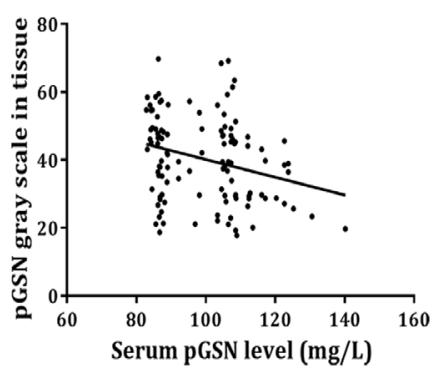

B

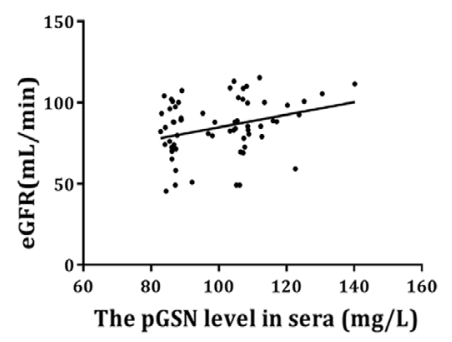

Fig. 5. Analysis of the correlations between the serum pGSN levels and both the pGSN levels in the glomerulus and the eGFR. (A) Analysis of the correlation between the serum pGSN levels and the pGSN levels in renal tissue $\left(\mathrm{r}=-0.2733,{ }^{*} p=0.0031\right)$. pGSN was specifically deposited in the glomerulus. (B) Analysis of the correlation with eGFR $\left(\mathrm{r}=0.3033,{ }^{*} p=0.0126\right)$. The correlations between the results were evaluated using Pearson's correlation. $p<0.05$ was considered significant. The total number of patients in each group $(\mathrm{n}=$ 200 ) was used for statistical analysis.

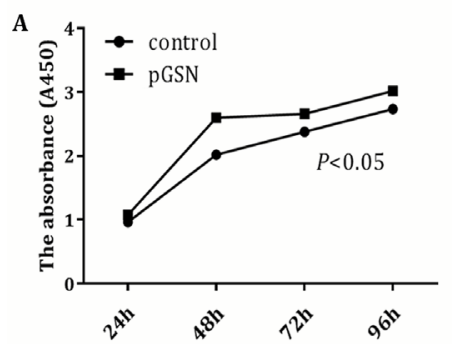

C

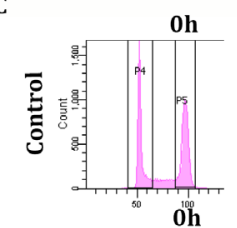

Oh
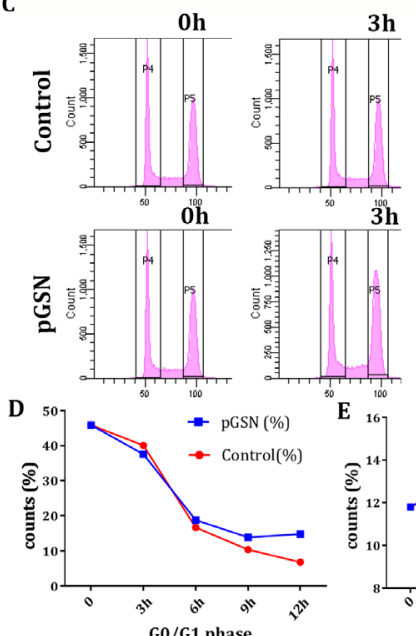

G0/G1 phase

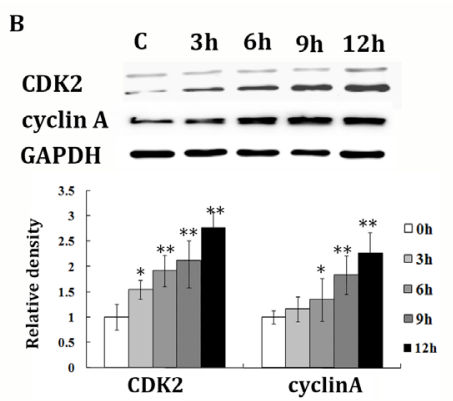

$6 h$
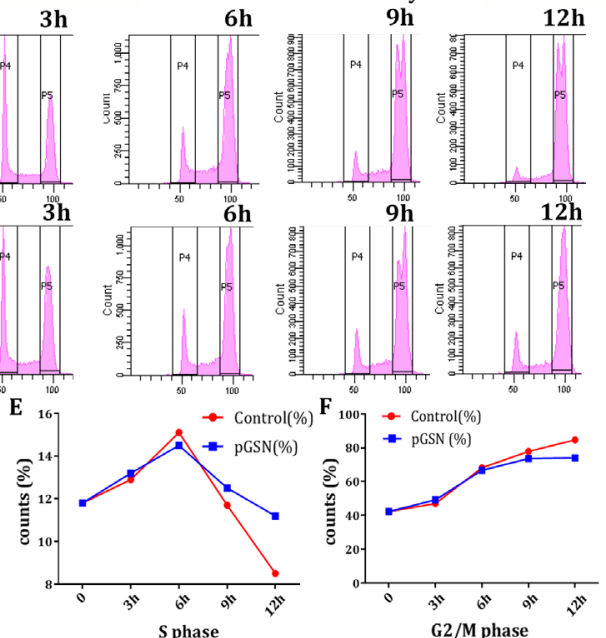

Fig. 6. pGSN promoted the proliferation of HMCs by facilitating cell mitosis. pGSN promoted the proliferation of HMCs $\left({ }^{*} p<0.05\right.$, two-way ANOVA). The HMCs were incubated with $10 \mathrm{mg} / \mathrm{L} \mathrm{pGSN}$ for $24 \mathrm{~h}, 48 \mathrm{~h}, 72 \mathrm{~h}$ or $96 \mathrm{~h}$. (B) Effects of pGSN on the expression of cell cycle regulatory proteins. MCs were treated with pGSN $(10 \mathrm{mg} / \mathrm{L})$ at $3 \mathrm{~h}, 6 \mathrm{~h}, 9 \mathrm{~h}$ or $12 \mathrm{~h}$. Western blot analysis showed the levels of CDK2 and cyclin A in MCs with or without pGSN treatment. (C-F) pGSN promoted the mitosis of HMCs. Cell cycle phases were detected in MCs that were treated with pGSN (10 mg/L) at $3 \mathrm{~h}, 6 \mathrm{~h}, 9 \mathrm{~h}$ or $12 \mathrm{~h}$. (C) Detection of the cell cycle in HMCs treated with or without pGSN. (D) Dynamic change in the percentage of cells in the G1 phase of the cell cycle in HMCs treated with or without pGSN at different time points. (E) Dynamic change in the percentage of cells in the $S$ phase of the cell cycle in HMCs treated with or without pGSN at different time points. (F) Dynamic change in the percentage of cells in the G2/M phase of the cell cycle in HMCs treated with or without pGSN at different time points. 


\section{Cellular Physiology Cell Physiol Biochem 2016;40:1473-1486

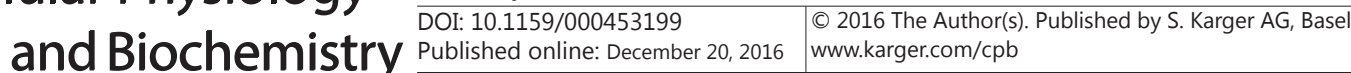 \\ Zhang et al.: The Role of pGSN in IgA Nephropathy}

in the G2/M phase decreased, which suggested that pGSN could promote the mitosis of HMCs (Fig. 6C, 6D-F). Furthermore, western blotting revealed that pGSN treatment resulted in a significant increase in the expression of CDK2 and cyclin A at different time points ( $3 \mathrm{~h}, 6 \mathrm{~h}, 9 \mathrm{~h}$ and $12 \mathrm{~h}$ ) and that pGSN also promoted the mitosis of HMCs in association with regulation of CDK2 and cyclin A expression (Fig. 6B, t-test, ${ }^{*} p<0.05,{ }^{* *} p<0.01$ ).

\section{Correlations between $p G S N$ and integrin} $\alpha 2 \beta 1$ expression in $\mathrm{HMCs}$

Integrins $\alpha 2 \beta 1$ were obviously up-regulated after pGSN treatment (Fig. 7A), and the integrin $\alpha 2 \beta 1-G S N$ interaction was enhanced after pGSN treatment or co-treatment with pGSN and aIgA1 (Fig. 7B).

\section{Discussion}

IgAN is the most common glomerular disease in the world. The pathological characteristics of IgAN include IgA immune complex deposition in the mesangium, glomerular MC proliferation, expansion of mesangial regions, massive ECM accumulation and renal fibrosis. MCs play important roles in renal physiological functions, inclu-

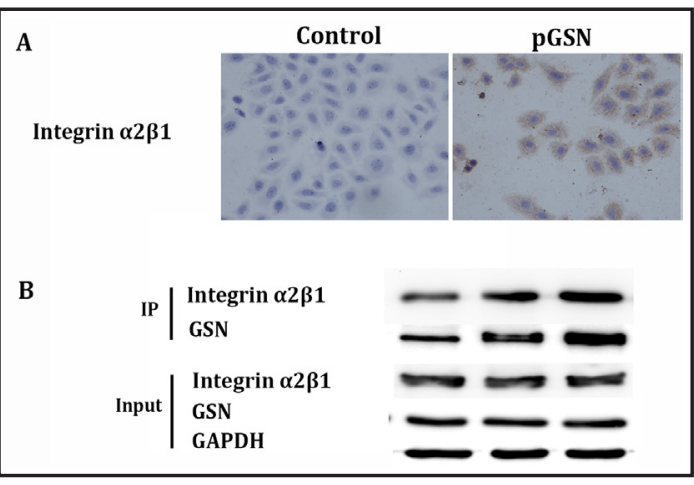

Fig. 7. pGSN promoted integrin $\alpha 2 \beta 1$ expression in HMCs and enhanced the integrin $\alpha 2 \beta 1$-GSN interaction. (A) pGSN increased integrin $\alpha 2 \beta 1$ expression in MCs. HMCs were treated with pGSN for $48 \mathrm{~h}$. The integrin $\alpha 2 \beta 1$ expression in MCs was stronger after pGSN treatment than in the absence of pGSN treatment, as determined using an immunohistochemical assay. (B) The integrin $\alpha 2 \beta 1$-GSN was enhanced after pGSN or aIgA1 treatment. HMCs were specifically treated with pGSN for $48 \mathrm{~h}$. The cells were then harvested, and whole extracts were immunoprecipitated with anti-GSN antibody. The immunocomplexes were analyzed by western blot analysis using antibodies against integrins $\alpha 2 \beta 1$ and GAPDH. In total, $20 \mu \mathrm{g}$ of each cell lysate (input) was used for SDS-PAGE followed by western blot analysis of GSN and integrin $\alpha 2 \beta 1$. GAPDH is shown as a loading control. ding by regulating intraglomerular capillary flow, phagocytosing and clearing foreign bodies, secreting cytokines [16], and generating ECM. However, excessive MC proliferation can lead to glomerular fibrosis. A lack of effective biomarkers limits the capacity to evaluate MC proliferation, glomerular fibrosis stages and IgAN progression, especially during the period before clinical symptoms become evident. Renal biopsy remains the gold standard for the assessment of the pathological degree in IgAN patients; nonetheless, this procedure is invasive and is associated with certain risks and sampling variabilities. In addition, the diagnostic accuracy of renal biopsy depends on the size and position of the biopsy specimen, and biopsy diagnoses made by different pathologists may be discordant. The present study represents the first proposal of potentially using pGSN as a biomarker for MC proliferation in IgAN patients. Specifically, our findings suggest the potential utility of pGSN as a prognostic indicator in IgAN.

GSN is an important actin-binding protein and a regulator of cellular cytoskeleton dynamics. In particular, pGSN is secreted into extracellular fluids. Serum pGSN levels decrease markedly in a variety of clinical conditions, such as lung injury [17], hepatitis B-associated liver cirrhosis [18], sepsis [19], major trauma[20], brain injury [21, 22], certain cancers [23, 24], Alzheimer's disease [25], rheumatoid arthritis [11] and multiple sclerosis [26]. Furthermore, certain studies have demonstrated that GSN is up-regulated in patients with idiopathic pulmonary fibrosis (IPF) or fibrotic nonspecific interstitial pneumonia [27, 28]. Another study found that the serum pGSN levels decreased in patients with hepatitis $B$ virus (HBV)-related cirrhosis and concluded that pGSN is a new candidate fibrosis marker in this condition [18]. However, the function of pGSN in IgAN has remained unclear.

Similarly, in our study, the serum pGSN levels were significantly reduced in the IgAN patients compared to the patients with other types of nephropathy and HCs. We also found 
that the pGSN levels were significantly higher in the kidneys of the IgAN group than in the kidneys of the MCG and HC groups. Furthermore, we found a negative correlation between the serum and kidney pGSN levels. Finally, we observed that pGSN promotes the proliferation of HMCs by facilitating cell mitosis in association with regulation of CDK2 and cyclin A expression, which in turn causes accumulation of the cytokines (such as TGF- $\beta$ ) secreted by HMCs and thus promotes the evolution of IgAN. These data indicated that serum pGSN levels correlate with MC proliferation and indirectly reflect glomerular fibrosis in IgAN.

In our study, the serum IgA and Gd-IgA1 levels in the IgAN patients were higher than those in the other groups. Moreover, the serum pGSN levels were negatively correlated with IgA in the IgAN patients, indicating that pGSN participates in the pathogenesis of IgAN. The above results suggest that IgA may interact with pGSN through an unknown mechanism. In addition to the transferrin receptor, integrins $\alpha 1 \beta 1$ and $\alpha 2 \beta 1$ are new candidate IgA1 receptors in human MCs [29], and GSN plays an important regulatory role in the integrin $\alpha 2 \beta 1$-mediated initial steps of collagen fibril attachment and engulfment in fibroblasts [30, 31]. To observe the receptors on HMCs treated with pGSN in the current study, integrins alpha1, 2/ beta1, 2 were detected by western blotting. Integrins $\alpha 2 \beta 1$ were obviously upregulated on the MCs after pGSN treatment, confirming the preferential interaction between GSN and integrins $\alpha 2 \beta 1$. We deduced that IgA1 and/or pGSN may combine with the integrin receptors to promote HMC proliferation and ECM production. We also added aIgA1 from IgAN patients to MCs to mimic the environment in IgAN, and the interaction between GSN and integrins $\alpha 2 \beta 1$ was remarkably strengthened after co-treatment with pGSN and aIgA1. This result further demonstrated that pGSN is easily deposited in the renal tissue in IgAN.

TGF- $\beta 1$ is a key factor in the pathogenesis of IgAN, and its levels correlate with the progress of IgAN [7]. The pathogenesis of TGF- $\beta 1 /$ Smad-mediated glomerular fibrosis has specifically been characterized [32]. The TGF- $\beta 1 /$ Smad signaling pathway adjusts the transcription and expression of its target genes, such as FN. FN is an important component of the ECM that exists as a soluble glycoprotein in the blood and other body fluids. The insoluble form of FN constitutes a major ECM protein in the course of glomerular fibrosis and sclerosis. In particular, the up-regulation of FN indicates excessive ECM deposition, eventually leading to glomerular sclerosis and renal fibrosis. In our study, the TGF- $\beta 1$ and FN levels in the sera and glomeruli were significantly higher than what would be associated with the degree of glomerular fibrosis. Moreover, the serum levels of TGF- $\beta 1$ and FN and the serum pGSN levels were negatively correlated, whereas the levels of these factors in the glomerulus were positively correlated. The pGSN levels in the glomeruli of the IgAN patients reflected the proliferation of the MCs that induce glomerular fibrosis.

In IgAN patients, the serum pGSN levels were significantly reduced and were positively correlated with the eGFR. Repeated attacks of gross or microscopic hematuria are common symptoms of IgAN; however, edema (76.84\%) is another indicator of IgAN that is often ignored, particularly in the early stages. In the present study, the pGSN levels in patients with edema were significantly decreased compared to the levels in patients without edema. A low pGSN level in the serum indicates serious renal injury, which could easily induce edema. The correlation between serum pGSN levels and several critical clinical conditions suggests the potential utility of pGSN as a prognostic marker.

In summary, our study revealed that the serum pGSN concentration reflected the progress and prognosis of IgAN; a low serum pGSN level specifically indicated a severe degree of proliferation of MCs and a worse prognosis. pGSN promotes the proliferation of MCs, thus contributing to IgAN progression, and the integrin $\alpha 2 \beta 1$ receptors may be the main binding sites for pGSN on MCs. Monitoring the pGSN levels in IgAN patients could thus help to predict prognosis and glomerular fibrosis and could be used to assess the progression of IgAN.

\section{Acknowledgments}

This work was supported by grants from the National Natural Science Foundation of China (No. 81300584, 81372611 and 81600539); the Chinese Postdoctoral Science Foundation 


\section{Cellular Physiology Cell Physiol Biochem 2016;40:1473-1486 \begin{tabular}{l|l|l} 
and Biochemistry Published online: & DOI: 10.1159/000453199 & $\begin{array}{l}\text { C 2016 The Author(s). Published by S. Karger AG, Basel } \\
\text { www.karger.com/cpb }\end{array}$
\end{tabular} \\ Zhang et al.: The Role of pGSN in IgA Nephropathy}

(2015M581472 and 2016T90310); the Postdoctoral Science Foundation of Heilongjiang Province of China (LBH-TZ0616); the Natural Science Foundation of Heilongjiang Province of China (LC2016038); the Technology Foundation for Selected Overseas Chinese Scholars, Heilongjiang Human Resources and Social Security Bureau (HongxueMeng); the Young Elite Training Foundation and HaiYan Scientific Research Foundation of Harbin Medical University Cancer Hospital (HongxueMeng); and the Harbin Science and Technology Innovation Fund (2016RAQXJ203).

\section{Disclosure Statement}

The authors have declared no conflict of interest.

\section{References}

1 Barratt J, Feehally J, Smith AC: Pathogenesis of IgA nephropathy. Semin Nephrol 2004;24:197-217.

2 D'Amico G: Natural history of idiopathic IgA nephropathy and factors predictive of disease outcome. Semin Nephrol 2004;24:179-196.

3 Imai H, Miura N: A treatment dilemma in adult immunoglobulin A nephropathy: what is the appropriate target, preservation of kidney function or induction of clinical remission? Clin Exp Nephrol 2012;16:195201.

4 Floege J: The pathogenesis of IgA nephropathy: what is new and how does it change therapeutic approaches? Am J Kidney Dis 2011;58:992-1004.

5 Leask A, Abraham DJ: TGF-beta signaling and the fibrotic response. FASEB J 2004;18:816-827.

6 Liu X, Hong Q, Wang Z, Yu Y, Zou X, Xu L: Transforming growth factor-beta-sphingosine kinase 1/S1P signaling upregulates microRNA-21 to promote fibrosis in renal tubular epithelial cells. Exp Biol Med (Maywood) 2016;241:265-272.

7 Bottinger EP, Bitzer M: TGF-beta signaling in renal disease. J Am Soc Nephrol 2002;13:2600-2610.

8 Schnaper HW, Jandeska S, Runyan CE, Hubchak SC, Basu RK, Curley JF, Smith RD, Hayashida T: TGF-beta signal transduction in chronic kidney disease. Front Biosci (Landmark Ed) 2009;14:2448-2465.

9 McGough AM, Staiger CJ, Min JK, Simonetti KD: The gelsolin family of actin regulatory proteins: modular structures, versatile functions. FEBS Lett 2003;552:75-81.

10 Li GH, Arora PD, Chen Y, McCulloch CA, Liu P: Multifunctional roles of gelsolin in health and diseases. Med Res Rev 2012;32:999-1025.

11 Osborn TM, Verdrengh M, Stossel TP, Tarkowski A, Bokarewa M: Decreased levels of the gelsolin plasma isoform in patients with rheumatoid arthritis. Arthritis Res Ther 2008;10:R117.

12 Working Group of the International Ig ANN, the Renal Pathology S, Cattran DC, Coppo R, Cook HT, Feehally J, Roberts IS, Troyanov S, Alpers CE, Amore A, Barratt J, Berthoux F, Bonsib S, Bruijn JA, D'Agati V, D'Amico G, Emancipator S, Emma F, Ferrario F, Fervenza FC, Florquin S, Fogo A, Geddes CC, Groene HJ, Haas M, Herzenberg AM, Hill PA, Hogg RJ, Hsu SI, Jennette JC, Joh K, Julian BA, Kawamura T, Lai FM, Leung CB, Li LS, Li PK, Liu ZH, Mackinnon B, Mezzano S, Schena FP, Tomino Y, Walker PD, Wang H, Weening JJ, Yoshikawa $\mathrm{N}$, Zhang H: The Oxford classification of IgA nephropathy: rationale, clinicopathological correlations, and classification. Kidney Int 2009;76:534-545.

13 Nakata J, Suzuki Y, Suzuki H, Sato D, Kano T, Yanagawa H, Matsuzaki K, Horikoshi S, Novak J, Tomino Y: Changes in nephritogenic serum galactose-deficient IgA1 in IgA nephropathy following tonsillectomy and steroid therapy. PLoS One 2014;9:e89707.

14 Wang Y, Zhao MH, Zhang YK, Li XM, Wang HY: Binding capacity and pathophysiological effects of IgA1 from patients with IgA nephropathy on human glomerular mesangial cells. Clin Exp Immunol 2004;136:168175.

15 Diven SC, Caflisch CR, Hammond DK, Weigel PH, Oka JA, Goldblum RM: IgA induced activation of human mesangial cells: independent of FcalphaR1 (CD 89). Kidney Int 1998;54:837-847. 


\section{Cellular Physiology Cell Physiol Biochem 2016;40:1473-1486

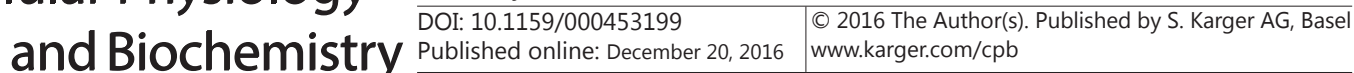 \\ Zhang et al.: The Role of pGSN in IgA Nephropathy}

16 Liang Y, Zhang J, Zhou Y, Xing G, Zhao G, Liu Z: Proliferation and Cytokine Production of Human Mesangial Cells Stimulated by Secretory IgA Isolated from Patients with IgA Nephropathy. Cell Physiol Biochem 2015;36:1793-1808.

17 DiNubile MJ: Plasma gelsolin levels in the diagnosis, prognosis, and treatment of lung complications of prematurity. Am J Respir Crit Care Med 2012;186:1195-1196.

18 Marrocco C, Rinalducci S, Mohamadkhani A, D'Amici GM, Zolla L: Plasma gelsolin protein: a candidate biomarker for hepatitis B-associated liver cirrhosis identified by proteomic approach. Blood Transfus 2010;8:s105-112.

19 Wang H, Cheng B, Chen Q, Wu S, Lv C, Xie G, Jin Y, Fang X: Time course of plasma gelsolin concentrations during severe sepsis in critically ill surgical patients. Crit Care 2008;12:R106.

20 Mounzer KC, Moncure M, Smith YR, Dinubile MJ: Relationship of admission plasma gelsolin levels to clinical outcomes in patients after major trauma. Am J Respir Crit Care Med 1999;160:1673-1681.

21 Zhao DQ, Wang K, Zhang HD, Li YJ: Significant reduction of plasma gelsolin levels in patients with intracerebral hemorrhage. Clin Chim Acta 2013;415:202-206.

22 Xu JF, Liu WG, Dong XQ Yang SB, Fan J: Change in plasma gelsolin level after traumatic brain injury. J Trauma Acute Care Surg 2012;72:491-496.

23 Pan S, Chen R, Brand RE, Hawley S, Tamura Y, Gafken PR, Milless BP, Goodlett DR, Rush J, Brentnall TA: Multiplex targeted proteomic assay for biomarker detection in plasma: a pancreatic cancer biomarker case study. J Proteome Res 2012;11:1937-1948.

24 Tsai MH, Wu CC, Peng PH, Liang Y, Hsiao YC, Chien KY, Chen JT, Lin SJ, Tang RP, Hsieh LL, Yu JS: Identification of secretory gelsolin as a plasma biomarker associated with distant organ metastasis of colorectal cancer. J Mol Med (Berl) 2012;90:187-200.

25 Guntert A, Campbell J, Saleem M, O'Brien DP, Thompson AJ, Byers HL, Ward MA, Lovestone S: Plasma gelsolin is decreased and correlates with rate of decline in Alzheimer's disease. J Alzheimers Dis 2010;21:585-596.

26 Kulakowska A, Ciccarelli NJ, Wen Q, Mroczko B, Drozdowski W, Szmitkowski M, Janmey PA, Bucki R: Hypogelsolinemia, a disorder of the extracellular actin scavenger system, in patients with multiple sclerosis. BMC Neurol 2010;10:107.

27 Whyte M: Gelsolin in idiopathic pulmonary fibrosis: a new target supports a central role for epithelial injury in disease pathogenesis. Thorax 2009;64:461-462.

28 Oikonomou N, Thanasopoulou A, Tzouvelekis A, Harokopos V, Paparountas T, Nikitopoulou I, Witke W, Karameris A, Kotanidou A, Bouros D, Aidinis V: Gelsolin expression is necessary for the development of modelled pulmonary inflammation and fibrosis. Thorax 2009;64:467-475.

29 Kaneko Y, Otsuka T, Tsuchida Y, Gejyo F, Narita I: Integrin alpha1/beta1 and alpha2/beta1 as a receptor for IgA1 in human glomerular mesangial cells in IgA nephropathy. Int Immunol 2012;24:219-232.

30 Arora PD, Janmey PA, McCulloch CA: A role for gelsolin in stress fiber-dependent cell contraction. Exp Cell Res 1999;250:155-167.

31 Arora PD, Glogauer M, Kapus A, Kwiatkowski DJ, McCulloch CA: Gelsolin mediates collagen phagocytosis through a rac-dependent step. Mol Biol Cell 2004;15:588-599.

32 Meng H, Zhang L, E X, Ye F, Li H, Han C, Yamakawa M, Jin X: Application of Oxford classification, and overexpression of transforming growth factor-beta 1 and immunoglobulins in immunoglobulin $\mathrm{A}$ nephropathy: correlation with World Health Organization classification of immunoglobulin A nephropathy in a Chinese patient cohort. Transl Res 2014;163:8-18. 